\title{
Compensatory Changes Accompanying Chronic Forced Use of the Nondominant Hand by Unilateral Amputees
}

\author{
Benjamin A. Philip ${ }^{1}$ and Scott H. Frey ${ }^{1,2}$ \\ ${ }^{1}$ Department of Psychological Sciences and ${ }^{2}$ Brain Imaging Center, University of Missouri, Columbia, Missouri 65211
}

\begin{abstract}
Amputation of the dominant hand forces patients to use the nondominant hand exclusively, including for tasks (e.g., writing and drawing) that were formerly the sole domain of the dominant hand. The behavioral and neurological effects of this chronic forced use of the nondominant hand remain largely unknown. Yet, these effects may shed light on the potential to compensate for degradation or loss of dominant hand function, as well as the mechanisms that support motor learning under conditions of very long-term training. We used a novel precision drawing task and fMRI to investigate 8 adult human amputees with chronic (mean 33 years) unilateral dominant (right) hand absence, and right-handed matched controls ( 8 for fMRI, 19 for behavior). Amputees' precision drawing performances with their left hands reached levels of smoothness (associated with left hemisphere control), acceleration time (associated with right hemisphere control), and speed equivalent to controls' right hands, whereas accuracy maintained a level comparable with controls' left hands. This compensation is supported by an experience-dependent shift from heavy reliance on the dorsodorsal parietofrontal pathway (feedback control) to the ventrodorsal pathway and prefrontal regions involved in the cognitive control of goal-directed actions. Relative to controls, amputees also showed increased activity within the former cortical sensorimotor hand territory in the left (ipsilateral) hemisphere. These data demonstrate that, with chronic and exclusive forced use, the speed and quality of nondominant hand precision endpoint control in drawing can achieve levels nearly comparable with the dominant hand.
\end{abstract}

Key words: amputation; behavior; deafferentation; fMRI; limb

\section{Introduction}

The contralateral hemisphere plays a primary role in controlling distal movements of the hand via crossed corticospinal pathways (for review, see Porter and Lemon (1993)). However, this system is functionally asymmetrical. Most humans strongly prefer the right hand in precision tasks (e.g., writing, drawing), which is widely held to reflect left hemisphere motor dominance (Liepmann, 1905; Coren and Porac, 1977; Geschwind et al., 2002; Sainburg, 2005; Shabbott and Sainburg, 2008; Jacobs et al., 2010). Exclusive use of the dominant hand $(\mathrm{DH})$ for precision tasks makes it difficult to distinguish the role(s) of intrinsic functional specializations from the effects of long-term practice. Lefthanded children who were forced to write with their nondominant hand $(\mathrm{NDH})$ continue to prefer the $\mathrm{DH}$ for other activities and therefore have not switched handedness (Siebner et al., 2002; Klöppel et al., 2007). However, amputees who have lost their

Received Sept. 3, 2013; revised Jan. 21, 2014; accepted Jan. 30, 2014.

Author contributions: B.A.P. and S.H.F. designed research; B.A.P. performed research; B.A.P. and S.H.F. analyzed data; B.A.P. and S.H.F. wrote the paper.

This work was supported by the United States Army Medical Research Acquisition Activity Grant W81XWH-092-0114 and National Institutes of Health/National Institute of Neurological Disorders and Stroke Grant NS083377 to S.H.F. We thank Sergei Bogdanov for his assistance with FSL; and Annaleigh Boggess, Noah Marchal, Julianna di Miceli, and Samantha Ross for their role in data collection.

The authors declare no competing financial interests.

Correspondence should be addressed to either Dr. Benjamin A. Philip or Dr. Scott H. Frey, University of Missouri, 1416 Carrie Francke Drive, 206 Melvin H. Marx Building, Columbia, M0 65211. E-mail: philipb@missouri.edu or freys@missouri.edu.

DOI:10.1523/JNEUROSCI.3770-13.2014

Copyright $\odot 2014$ the authors $\quad 0270-6474 / 14 / 343622-10 \$ 15.00 / 0$ right $\mathrm{DH}$ are forced to exclusively and chronically use their left NDH. Therefore, amputees provide a unique opportunity to evaluate the effects of experience on hand dominance.

In addition to contralateral responses, unilateral upper extremity amputees show robust ipsilateral cortical activity when moving their intact hands (Kew et al., 1994; Hamzei et al., 2001), specifically in the former sensorimotor hand territory (Bogdanov et al., 2012). However, the functional role of this response, if any, remains unknown. One possibility is that decreased activity contralateral to injury leads to decreased interhemispheric inhibition (Werhahn et al., 2002a; Pelled et al., 2009; Pawela et al., 2010). Alternatively or additionally, this increased bilateral activation in amputees may reflect a functional adaptation to long-term forced use of a single hand (Elbert and Rockstroh, 2004; Frey et al., 2008). This dovetails with recent findings that ipsilateral motor cortex plays a functional role in NDH learning and performance among healthy adults (Verstynen et al., 2005; Vines et al., 2008; Schambra et al., 2011).

A recent behavioral study of $\mathrm{NDH}$ writing by $\mathrm{DH}$ amputees found pronounced variability across individuals and tasks (Yancosek and Mullineaux, 2011). However, because writing is highly individualized, it is difficult to compare the accuracy of NDH performances with estimates of controls' $\mathrm{DH}$ performances across participants. To address this issue, we developed a novel precision drawing task (PDT), with the intent of capturing the precision demands of naturalistic handwriting and drawing.

We used the PDT to test two hypotheses. First, if right hand dominance for precision control reflects intrinsic and unalterable 
Table 1.Demographic data on amputee participants ${ }^{a}$

\begin{tabular}{|c|c|c|c|c|c|c|c|c|}
\hline Age (years) & Sex & Above/below elbow & YSA & Prosthesis & Dominant right hand $(0-1)$ & Current pain $(0-1)$ & Current phantom presence $(0-1)$ & Current phantom mobility $(0-1)$ \\
\hline 56 & Male & Above & 33 & None & 1 & 0.25 & 0.69 & 0.01 \\
\hline 66 & Male & Below & 44 & Mechanical & 0.98 & 0 & 0 & 0 \\
\hline 73 & Male & Above & 8 & None & 0.93 & 0.09 & 0.11 & 0.11 \\
\hline 62 & Male & Above & 25 & None & 1 & 0.04 & NA & 0 \\
\hline 55 & Male & Below & 38 & None & 0.98 & 0.01 & 0.3 & 0.2 \\
\hline 67 & Male & Below & 46 & None & 0.98 & 0.29 & 0.813 & 0.8 \\
\hline 62 & Male & Below & 41 & Mechanical & 1 & 0 & 0 & 0 \\
\hline 52 & Female & Above & 31 & None & 0.98 & 0.83 & NA & 0.99 \\
\hline
\end{tabular}

${ }^{a}$ Prosthesis type measured by usage in the past 5 years; 0 -1 measurements determined by visual analog scale before study. "Dominant right hand" question phrased as, "Before my amputation, I always used my right hand for writing and drawing." YSA, Years since amputation; NA, not available.

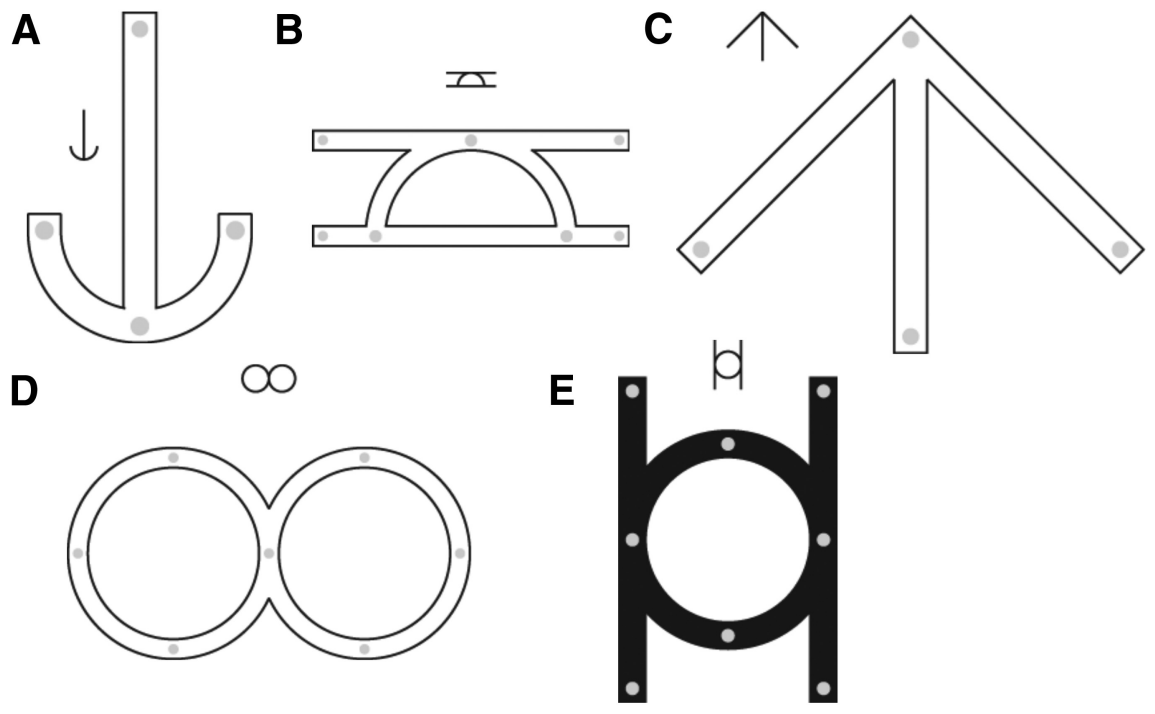

Figure 1. Sample precision drawing task (PDT) stimuli, showing 5 of 15 possible shapes. $A, 90 \mathrm{~mm}$ drawing length, $5 \mathrm{~mm}$ width. $\boldsymbol{B}, 135 \mathrm{~mm}$ drawing length, $3 \mathrm{~mm}$ width. $\boldsymbol{C}, 135 \mathrm{~mm}$ drawing length, $5 \mathrm{~mm}$ width. $\boldsymbol{D}, 180 \mathrm{~mm}$ drawing length, $3 \mathrm{~mm}$ width. $\boldsymbol{E}$, Vision-control stimulus with $180 \mathrm{~mm}$ drawing length, $4 \mathrm{~mm}$ width.

specializations (e.g., of the left cerebral hemisphere), then amputees' NDH performances should never achieve the level of controls' DH performances, even after chronic forced use. Alternatively, if the right hand advantage for precision control is experience-dependent, then chronic forced use could allow amputees' NDH performances to achieve levels comparable with matched controls' DHs. This could arise from training-related changes in the right hemisphere (contralateral to NDH) and/or increased recruitment of the left dominant hemisphere (Sainburg, 2010). Second, if the former sensorimotor hand territory contributes to NDH functional compensation, then activity within this region during the PDT will be related to amputees' $\mathrm{NDH}$ performances.

\section{Materials and Methods}

\section{Participants}

Eight unilateral upper-limb traumatic amputees (1 female, 4 belowelbow, mean age $62 \pm 7$ years, age range $52-73$ years, mean time since amputation $33 \pm 12$ years) and 8 controls matched for age and gender gave informed consent to participate in this study. An additional 11 controls gave informed consent for behavioral participation, creating a group of 19 behavioral controls ( 3 female, mean age $63 \pm 7$ years, age range 53-73 years). One additional behavioral participant was removed from the analyses because he developed a visual disorder between sessions. All amputees were right-hand dominant with right-side amputations that occurred no earlier than age 17. All controls were right-hand dominant (Edinburgh score $>60$ ) (Oldfield, 1971). See Table 1 for details on the amputee sample. All procedures were approved by the local institutional review board and in accordance with the Declaration of Helsinki.

\section{Experimental design overview}

To summarize, each participant performed an initial behavioral testing session, followed by an fMRI session later in the day. During each session, participants performed a PDT, in which they drew continuous lines within provided boundaries using their left hands. Controls returned for a second set of sessions using their right hands, 6-53 d (24 $\pm 18 \mathrm{~d})$ later; order of hands was counterbalanced across participants. For purposes of direct comparison with amputees, we report fMRI data only from the NDH (left hands) for both groups.

\section{PDT}

To perform the PDT, participants used a pencil to draw within the boundaries of a geometric form (Fig. $1 A-D$ ). All stimuli were mirrorsymmetrical, to avoid any possible interactions between intermanual transfer processes and mirror-reversing movements. Stimuli comprised combinations of 2-4 simple geometric elements: horizontal lines, vertical lines, diagonal lines, and semicircles. A single element (line or semicircle) had a drawing length of $45 \mathrm{~mm}$; therefore, a stimulus could have drawing length of $90-180 \mathrm{~mm}$. Stimuli could have width of 3 , 4 , or $5 \mathrm{~mm}$. A "stimulus" refers to a unique combination of elements and width; a "shape" refers to the same combination of elements at any width. In other words, if two stimuli were identical except for their width, they were both the same shape. The total set of possible PDT shapes were 5 stimuli comprising 2 elements ( $90 \mathrm{~mm}), 5$ stimuli comprising 3 elements $(135 \mathrm{~mm})$, and 5 stimuli comprising 4 elements $(180 \mathrm{~mm})$. Therefore, there were 15 possible shapes, leading to 30 stimuli in the fMRI session (15 shapes $\times 2$ widths) and 45 stimuli in the behavioral session (15 shapes $\times 3$ widths).

A "trial" refers to the presentation and completion of one stimulus during the behavioral session. The behavioral session entailed 90 trials (2 of each stimulus). PDT performance was only analyzed from the behavioral session because of the difficulty of online data collection during fMRI.

Participants were instructed to complete as many stimuli as possible within the allotted time, without making any errors; they were told to favor precision over speed. A trial could have one of three possible labels: successful, error, or incomplete. A trial was considered an "error" (10.7\% of all trials) if the drawn lines ever passed outside the stimulus' margin, except for overshoots at the end of segments and for distinct accidental lines created as part of raising or lowering the pen. A trial was considered "incomplete" ( $0.4 \%$ of all trials) if the drawn lines did not pass fully through all open paths within the stimulus. A trial was considered a "success" if it was neither an error nor incomplete. Both successful and error trials were used for further analyses to maintain trial counts across conditions; however, removing error trials led to negligible effects on the 
data and no changes in conclusions. Also, if a trial's speed exceeded \pm 3 SDs from the mean (calculated separately for each participant and stimulus width), then it was considered an outlier and not included in the movement speed analysis.

Ratings of trial status were performed by hand. At least two different experimenters examined each participant's drawings to analyze inter-rater reliability. The two most frequent raters had an agreement rate of $0.94 \pm 0.03$ across sessions and an inter-rater reliability (Cohen's $\kappa$ ) of $0.75 \pm 0.13$. Ratings from the most frequent and senior experimenter were used for analysis.

Performance was measured by calculating "mean speed" ( $\mathrm{mm} / \mathrm{s})$ for each successful trial. Error rates were calculated for each session as the number of errors divided by the number of completed trials. For analysis of possible practice or fatigue effects, trials were separated into three epochs: early (trials 1-30), middle (31$60)$, and late (61-90).

\section{Behavioral session and training}

During each behavioral session, participants performed the PDT on a Cintiq $12 \mathrm{wx}$ tablet with integrated $1200 \times 800$ screen $($ Wacom), controlled by Presentation software, version 14.9 (Neurobehavioral Systems). The participants were presented with PDT forms in pseudorandom order. After the participant completed each trial, the experimenter pressed a button to advance to the next trial. The participants performed 90 trials, 2 of each stimulus, and we collected endpoint speed and position via the tablet. These behavioral data collection trials were performed in a seated position, with the tablet on a table in front of the participant. All participants were instructed to not move their forearm, and control the pen only with their fingers and wrist. This was done to prepare for later fMRI testing in which shoulder movements produce head motion and associated artifacts.

After behavioral data collection, MRI participants received 20 min of training in a "fMRI simulator," which replicated the physical constraints of a fMRI system, without a magnet. During the simulator training, participants performed the $\mathrm{fMRI}$ version of the PDT, as described below.

\section{Behavioral data analysis}

The tablet collected $x$ and $y$ position of the pen endpoint at $30 \mathrm{~Hz}$. During analysis, velocity profiles for each trial were smoothed using an acausal Gaussian filter with mean of 3 samples (100 ms).

Four dependent measures were analyzed: speed $(\mathrm{mm} / \mathrm{s}$, mean of each trial), endpoint smoothness (positive values indicating fewer submovements: calculated as $-1 \times$ number of velocity peaks per $45 \mathrm{~mm}$ element, mean of each trial), acceleration time (fraction of time spent with acceleration $>0$, mean of each trial), and error rate (number of trials with errors/number of completed trials, mean of a session). These four variables were chosen to capture movement characteristics specialized to either hand (Mutha et al., 2012), including goal-directed performance (via speed and error), acceleration/distance relations across multistage movements (via acceleration time), and arm dynamics (via smoothness).

Each of the four measures was analyzed using a three-way betweengroups ANOVA, including the factors: group (Amp-NDH, Ctl-NDH, Ctl-DH), stimulus width $(3,4,5 \mathrm{~mm})$, and epoch (early, middle, late). Post hoc analyses were performed using Tukey's HSD, with power analyses performed using MATLAB's sampsizepwr function (two-tailed $t$ test, $\alpha=0.05)$. All behavioral analyses were performed with MATLAB 8.10 (MathWorks).

\section{fMRI session}

PDT task design. During the fMRI session, participants lay in an MRI scanner in the position described in Figure 2A. Participants used a video camera to observe a display apparatus in their lap and used a pencil to draw on paper contained within the apparatus (Fig. $2 B$ ). The display apparatus allowed the participant to use a pencil to perform the PDT on paper and also allowed a rapid transition between stimuli without permitting advance view of upcoming stimuli. After completing each figure, participants closed their eyes and placed their pencil on the apparatus frame outside of the drawing space (the "ready position"; Fig. 2B). At that time, the experimenter removed the current page from the apparatus, which revealed the page below it. Then, the experimenter immediately touched the participant's arm, thereby directing the participant to reopen their eyes and continue. In this way, participants had their eyes closed between stimuli, rather than seeing the paper withdrawn from the apparatus. These steps were repeated until the end of the block was reached.

At the end of each block, a "reload period" occurred in which the experimenter replaced the current apparatus with a new apparatus containing stimuli for the subsequent block. Participants were instructed to close their eyes during the reload period, and the video feed was replaced with a static image. Figure $2 C$ provides the timeline for events within a run.

The PDT included four alternating conditions in a block design: "Task-Easy" (5 mm width), "Task-Hard" (3 mm width), "FixationRest," and "Vision-Control." During the two task conditions, the participant performed the PDT as described above. During the Fixation-Rest condition, the paper in the apparatus contained only a fixation cross, throughout the entire block. During the Vision-Control condition, the paper in the apparatus showed PDT outlines ( $4 \mathrm{~mm}$ width) with no blank space to draw in (Fig. 1E). During these Vision-Control blocks, participants touched their pencil to the stimulus and observed the resulting static combination of stimulus, pencil, and hand. At the end of the stimulus (5-14 s, to approximate the rate of stimulus change during task performance), the participants put their pencil in the ready position and closed their eyes. Subsequently, the experimenter presented a fresh stimulus, as described above.

A 37 min session comprised 6 runs of 6.12 min each. Each run comprised 12 blocks of $20.4 \mathrm{~s}$ each, a $7.65 \mathrm{~s}$ reload period after each block, and a single 30.6 s eyes-closed rest period ("Closed-Rest") at the end of the run. The 12 blocks in each run comprised three instances of each of the four conditions; the experiment always alternated between control 
conditions and task conditions, but the orders of the two control conditions (Fixation-Rest and Vision-Control) and the two task conditions (Task-Easy and Task-Hard) were counterbalanced across runs. Counterbalancing also ensured that the possible transitions between blocks (e.g., Task-Hard immediately followed by FixationRest) occurred with equal frequency; 8 each of the 8 transition types accounted for 64 of the 66 transitions, with the last two chosen randomly. In total, the main fMRI session entailed 18 instances of each condition, adding up to $6.12 \mathrm{~min}$ of each condition. The Closed-Rest condition occurred in 6 instances, for a total of $3.06 \mathrm{~min}$.

Within each run, the stimuli were presented in pseudorandom order, separately for each condition, such that each stimulus could only appear once per run at most.

For control participants, the unused hand was restrained with a sand bag weight, to prevent them from using the hand or arm for any purpose (e.g., stabilizing the apparatus).

Localizer task design. After the main fMRI session described above, participants performed a novel precision drawing localizer task designed to identify all brain areas involved in visually guided drawing movements. Localizer stimuli were presented in the same manner as the main task stimuli. However, during the localizer task, the stimuli were an array of 12-20 randomly placed dots, unique for every trial. Participants were instructed to use the pencil to "connect the dots" in any pattern that would link all the dots. This task was chosen to activate a broad variety of areas involved in making goal-oriented drawing movements under visual guidance.

The localizer task alternated between task ("Localizer-Task") blocks and rest blocks during which the eyes remained closed ("Rest"). At the end of every block, the video feed was replaced with a fixed "close image," and a reload period occurred. At the start of a Localizer-Task block, the experimenter touched the participant's arm, thereby signaling the participant to open their eyes. Otherwise (during Rest blocks) their eyes remained closed. The "close image" remained on screen throughout the Localizer-Rest blocks.

A 12 min session comprised 3 runs of 5.6 min each. Each run comprised 12 blocks (6 Localizer-Task and 6 Rest in alternating order) of $20.4 \mathrm{~s}$ each, plus a $7.65 \mathrm{~s}$ "reload period" after each block. The choice of which block to present first was counterbalanced across runs. In total, this entailed 18 instances of each condition, adding up to $6.12 \mathrm{~min}$ of each condition.

Scanning procedure. Scans were performed on a Siemens 3T Magnetom Allegra. BOLD EPIs were collected using a T2*-weighted gradient echo sequence, a standard birdcage radio-frequency coil, and the following parameters: $\mathrm{TR}=2550 \mathrm{~ms}, \mathrm{TE}=30 \mathrm{~ms}$, flip angle $=80^{\circ}, 64 \times 64$ voxel matrix, FOV $=200 \mathrm{~mm}, 42$ contiguous axial slices acquired in interleaved order, thickness $=4.0 \mathrm{~mm}$, in-plane resolution: $3.1 \times 3.1$ $\mathrm{mm}$, bandwidth $=2604 \mathrm{~Hz} /$ pixel. PACE (Thesen et al., 2000) was used for online motion correction. The initial 2 volumes in each scan were discarded to allow steady-state magnetization to be approached. Siemens auto-align was run at the start of each session.

High-resolution T1-weighted structural images were also acquired, using the 3D MP-RAGE pulse sequence: $\mathrm{TR}=2500 \mathrm{~ms}, \mathrm{TE}=4.38 \mathrm{~ms}$, $\mathrm{TI}=1100 \mathrm{~ms}$, flip angle $=8.0^{\circ}, 256 \times 256$ voxel matrix, FOV $=256 \mathrm{~mm}$, 176 contiguous axial slices, thickness $=1.0 \mathrm{~mm}$, in-plane resolution: $1.0 \times 1.0 \mathrm{~mm}$.

DICOM image files were converted to NIFTI format using MRIConvert software (http://lcni.uoregon.edu/ jolinda/MRIConvert/).

\section{Preprocessing}

Structural and functional fMRI data were preprocessed and analyzed using fMRIB's Software Library (FSL version 4.1.8; http://www.fmrib.ox. ac.uk/fsl/) (Smith et al., 2004) and involved several steps: Nonbrain structures were removed using BET. The data were spatially smoothed using a Gaussian kernel of $5 \mathrm{~mm}$ FWHM. For each dataset, intensity normalization was applied using "grand mean scaling," wherein each volume in the dataset was scaled by the same factor to allow for valid cross-session and cross-subject statistics. A high-pass temporal filter with $100 \mathrm{~Hz}$ cutoff was applied to remove low-frequency artifacts. Independent components analysis was conducted with MELODIC to denoise the data, following procedures detailed below. Time series statistical analysis was performed in FEAT version 5.98 using FILM with local autocorrelation correction (Woolrich et al., 2001). Delays and undershoots in the hemodynamic response were accounted for by convolving the model with a double-gamma HRF function. Functional data were registered with the high-resolution structural image with 7 degrees of freedom, and resampled to $2 \times 2 \times 2 \mathrm{~mm}$ resolution using FLIRT; the participant images were then registered to standard images (MNI-152) using FNIRT nonlinear registration.

MELODIC independent components analysis was used to identify artifactual components for removal. Components were rejected if they clearly met one of the following criteria: (1) preponderance of suprathreshold voxels in nonbrain areas, including ventricles or a "halo" outside the brain; (2) spin history effects, specifically activations that appear in alternating slices; (3) absence of peaks in the lower-frequency half of the power spectrum; (4) time course dominated by 1 or 2 distinct temporal spikes; (5) components covering the majority of 1 or 2 slices, but not neighboring slices; and (6) Nyquist ghosts. Only the 20 components with the highest contributions were tested for rejection; $16.2 \pm 2.3$ components were rejected per run, accounting for $35.0 \%$ of all components.

\section{Localizer task $f M R I$ analysis}

To identify all areas involved in visually guided drawing, we first analyzed the three localizer runs. An explanatory (predictor) variable (EV) was modeled, along with its temporal derivatives, for the Localizer-Task condition. Time with the eyes closed (Rest and reload periods) was left unmodeled and served as the implicit baseline. EVs were locked to the onset of the camera visual feed at the start of each block. Orthogonal contrasts (one-tailed $t$ tests) were used to test for differences between the experimental condition and baseline.

First-level contrasts of parameter estimates (COPEs) were calculated for the Localizer-Task condition. The first-level COPEs served as input to a second-level analysis, which averaged the 3 runs using a fixed-effects model. $Z$-statistic (Gaussianized T) images were thresholded using clusters specified by $Z>4.1$ (corrected cluster significance threshold $p=$ 0.05 ); changing the $Z$ threshold did not substantially change the mask. Voxels with significantly increased activity for this contrast (LocalizerTask relative to baseline) were used as an inclusive mask for PDT task analysis.

\section{PDT task fMRI analysis}

Analysis of PDT data was restricted to voxels that demonstrated significant increases in activity during the localizer task compared with rest, as described in the preceding section.

EVs were modeled, along with their temporal derivatives, for the following five conditions: Task-Easy, Task-Hard, Task (i.e., "Task-Any," including both Task-Easy and Task-Hard periods), Vision-Control, and Fixation-Rest. Time with the eyes closed (Closed-Rest and reload periods) was left unmodeled and served as the implicit baseline. EVs were locked to the onset of the camera visual feed at the start of each block. Orthogonal contrasts (one-tailed $t$ tests) were used to test for differences between each experimental condition.

First-level COPEs were calculated for each of the five conditions listed above and also for Task versus Vision-Control. For this latter contrast, prethreshold masking as used to include only voxels that showed significant activation during both conditions, to ensure that any results would not be attributable to differences in relative deactivation. Likewise, we contrasted Task-Hard versus Task-Easy (masked to include only voxels with positive activation for both Task-Easy and Task-Hard). The firstlevel COPEs served as input to higher-level analyses performed using a fixed-effects model. $Z$-statistic (Gaussianized T) images were thresholded using clusters determined by $Z>3.1$ and a corrected cluster significance threshold of $p=0.05$. The first-level COPEs were averaged across runs for each subject separately (second level) and then across participants (third level). At the third level, direct comparisons were conducted between groups by modeling two EVs, one for each group. In between-groups analyses, inclusive masking restricted analysis to areas with positive activation for at least one group (amputees or controls). During all third-level analyses, $Z$-statistic images were thresholded using 
clusters determined by $Z>3.1$. Reducing the threshold to 2.3 did not substantially change the images. Because of the limited sample size inherent in studying right-hand-dominant, right-limb-loss, MRI-compatible amputees, a fixed-effects analysis was used for third-level analyses.

Multifiducial mapping in Caret version 5.64 (http://www.nitrc.org/projects/caret/) was used to overlay group statistical maps onto a population-average, landmark- and surfacebased (PALS) atlas for visualization (Van Essen, 2005). Slice views were produced using Mricron version 12/2009 (http://www.mccauslandcenter. sc.edu/mricro/mricron/).

\section{Sensorimotor hand territory functional ROI analysis}

Normative sensorimotor hand representations were defined using data from a functional localizer task in which an independent sample of 17 healthy adults (mean age 52 years, range 29-62 years, 4 female, 1 lefthanded) performed an aurally paced, thumb-finger sequencing task with eyes closed (Smith and Frey, 2011). Contrasts between hand movement versus rest yielded two significant regions of increased activity in contralateral sensorimotor cortex: one for left hand movement and one for right hand movement, that is, in the right hemisphere (RHem) and left hemisphere (LHem). Spherical ROIs (5 $\mathrm{mm}$ radius) were centered on the peak activations in these two regions, at $X=-38, Y=-24, Z=54$ (left hemisphere) and $X=38, Y=-24, Z=54$ (right hemisphere) in MNI-152 space. Inverse registration with FLIRT was used to transform the initial standard-space ROIs into the high-resolution structural images for each participant in this study, producing participantspecific ROIs.

Mean percentage signal change (PSC) between the Task condition and baseline (Vision-Control) was calculated across all voxels separately within each ROI, for each participant, using FSL's Featquery. We then submitted mean PSC values to a 2 (Group: amputee, control) $\times 2$ (Hemisphere: left, right) repeated-measures ANOVA. Power analyses for correlation values were performed via Monte Carlo simulations using 10,000 draws to establish chance values, and 1000 draws from the data at each tested sample size (up to sample sizes of $N=500$ ) to establish the sample size needed to detect significance with $80 \%$ power.

\section{Results}

\section{Behavioral performance}

Our primary dependent measures were movement speed, movement smoothness, fraction of time spent accelerating, and error rate. To evaluate the effects of chronic amputation and task difficulty on PDT behavior, we performed 3 (stimulus width) $\times 3$ (epoch) $\times 3$ (group) ANOVAs on each dependent variable.

For movement speed, we found main effects of Group $\left(F_{(2,395)}\right.$ $=38.94, p<0.0001)$ and Width $\left(F_{(2,395)}=19.47, p<0.0001\right)$, but no main effect of Epoch $(p>0.19)$ and no significant interaction effects $(F<1.0)$. Post hoc tests revealed that the main effect of width arose from steadily increasing speed for wider stimuli that required less precision, as illustrated in Figure $3 A$ (all widths
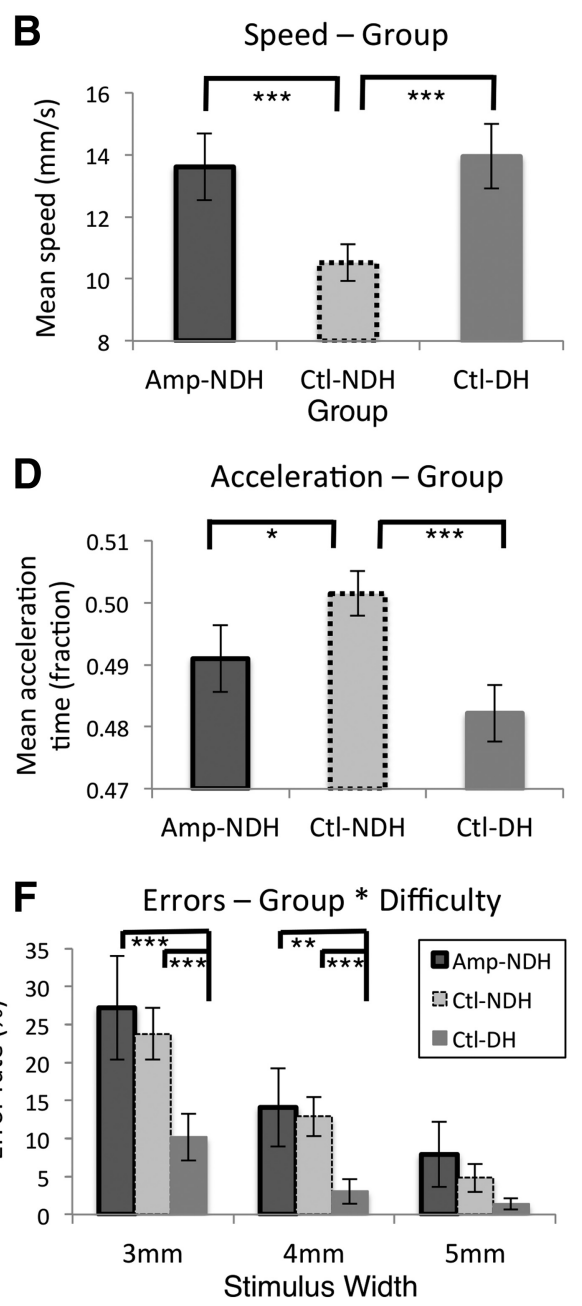

Figure 3. PDT performance during behavioral session, showing that Amp-NDH performance greatly improved from Ctl-NDH performance. Group means \pm SEM. ${ }^{*} p<0.05 .{ }^{* *} p<0.01{ }^{* * *} p<0.001 . \boldsymbol{A}$, Effect of task difficulty on speed. Easier task (wider

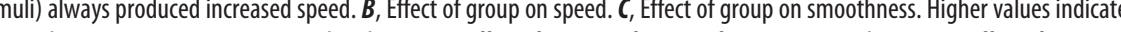
ents $45 \mathrm{~mm}$ stimulus element. $\boldsymbol{D}$, Effect of group on fraction of time spent accelerating. $\boldsymbol{E}$, Effect of group on error rate. $\boldsymbol{F}$, Interaction between group and task difficulty, on error rate; group effect occurs on narrow $(3 \mathrm{~mm})$ and medium (4 $\mathrm{mm}$ ) widths only. Main effects of group not shown.

significantly different at $p<0.01)$. The main effect of group arose from lower speed for the Ctl-NDH group compared with Ctl-DH or Amp-NDH $(p<0.0001)$, as shown in Figure $3 B$.

For movement smoothness, we again found main effects of $\operatorname{Group}\left(F_{(2,395)}=37.66, p<0.0001\right)$ and Width $\left(F_{(2,395)}=15.87\right.$, $p<0.0001)$, but no main effect of Epoch $(p>0.14)$ and no significant interaction effects $(F<1.0)$. Post hoc tests revealed that the main effect of group arose from lower smoothness for the Ctl-NDH group compared with Ctl-DH or Amp-NDH, as shown in Figure $3 C(p<0.0001)$. The main effect of width arose from lower smoothness for the narrowest stimuli $(3 \mathrm{~mm})$ compared with wider stimuli ( $p<0.01$ vs $4 \mathrm{~mm}, p<0.0001$ vs $5 \mathrm{~mm}$ ).

For acceleration time, we found a main effect of Group $\left(F_{(2,395)}=21.4, p<0.0001\right)$ but no other significant main or interaction effects $(p>0.2)$. Post hoc tests revealed that the main effect of group arose because Ctl-NDH spent more time accelerating than other groups ( $p<0.05$ vs Amp-NDH, $p<0.0001$ vs Ctl-DH), as shown in Figure $3 D$. Amp-NDH showed a nonsignificant trend toward increased acceleration time compared with Ctl-DH $(p=0.065)$. Power analysis indicated that the difference in acceleration time between Amp-NDH and Ctl-DH would 


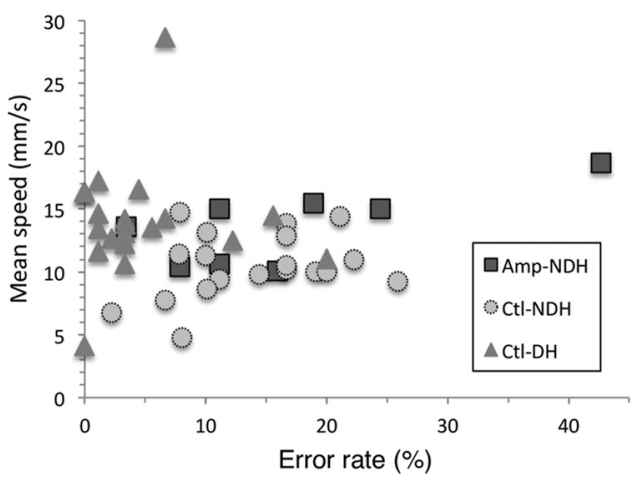

Figure 4. PDT performance during behavioral session, comparing individual mean speed and error rate for each participant. There were no significant speed-accuracy relationships found for any group, after accounting for outlier (participant with $42 \%$ error; see Results).

reach statistical significance with 92 participants at the current effect size.

For error rate, we again found main effects of Group $\left(F_{(2,395)}=37.88, p<0.0001\right)$ and Width $\left(F_{(2,395)}=57.05, p<\right.$ $0.0001)$, a nonsignificant trend toward a main effect of Epoch $\left(F_{(2,395)}=2.72, p=0.067\right)$, and a statistically significant interaction between Group and Width $\left(F_{(4,395)}=3.5, p<0.01\right)$, but no other interaction effects $(F<1.0)$. The main effect of group arose from fewer errors for the Ctl-DH group compared with either Ctl-NDH or Amp-NDH $(p<0.0001)$, as shown in Figure $3 E$. The main effect of width arose from steadily increasing error rates for narrower stimuli that required more precision (all widths significantly different at $p<0.01$ ). The nonsignificant trend in Epoch reflected elevated error rates in the first epoch, compared with other epochs. Finally, the Group $\times$ Width interaction arose because Ctl-DH group had lower error rates than both Ctl-NDH and Amp-NDH groups for narrow $(3 \mathrm{~mm})$ and medium $(4 \mathrm{~mm})$ stimuli, but the groups did not differ for wide $(5 \mathrm{~mm}$ ) stimuli (all comparisons significantly different at $p<0.01$ ), as shown in Figure $3 F$.

Figure 4 shows the individual mean PDT speed and error rate for each participant. Across all participants, we found no statistically significant correlation between individual mean speed, and individual error rate $(r=0.050, p=0.744)$. Likewise, speed and error rate were not significantly correlated for controls' DH $(r=0.047, p=0.850)$ or NDH $(r=0.305, p=0.205)$ performances. For amputees, we found a marginally significant positive correlation between speed and error rate $(r=0.715, p=0.046)$. However, this apparent correlation was driven by the outlier participant on the far right side of Figure 4 (42\% error rate); without that participant, Amp-NDH $(r=0.313, p=0.494)$. Thus, after accounting for the outlier, we found no speed-accuracy relationships for any group.

Finally, we found no statistically significant correlations between speed or error rate and any amputee characteristic: (age, years since amputation [YSA]), phantom pain, phantom presence, phantom mobility) ( $p>0.08$ in all cases). Our minimum YSA was 8 , so any potential YSA effect could have reached plateau for all participants.

In summary, of our four kinematic measurements, three showed complete compensation of NDH to DH levels of performance after amputation (speed, smoothness, and acceleration time), and one showed no compensation (error rate). Overall, these data support the hypothesis that, after chronic (mean 33 years) forced use, the speed and quality of amputees' precision drawing movements with the NDH are greatly improved and closely approximate levels of performance that would have been expected with their DHs. We now turn to the neural mechanisms supporting this remarkable compensation.

\section{fMRI results}

Here we focus on data from amputees and controls using their $\mathrm{NDH}$ only.

\section{Effects of task difficulty}

A comparison between Task-Hard versus Task-Easy failed to detect any areas sensitive to difficulty. For subsequent analyses, we therefore pooled data across these two conditions (i.e., "TaskAny" condition).

\section{Precision drawing engages distributed sensorimotor networks in} controls and amputees

To provide an overview of brain activation during performance of the PDT by healthy controls using their NDHs (left hands), we identified areas showing increased activity for Task compared with Vision-Control, within the visuosensorimotor mask defined from our localizer task. This analysis revealed increased bilateral activity within the insular cortex and throughout the dorsal sensorimotor processing stream: extending from the occipital lobes through the parietal, motor, and into premotor cortices. Increases were also apparent the midline in the supplementary and cingulate motor areas, and subcortically in the thalamus, putamen, globus pallidus, and the cerebellar vermis and dorsal hemispheres (Fig. 5A). We obtained similar results for amputees using their NDHs (Fig. 5B).

Relative to amputees, controls showed increased activity primarily within the frontoparietal dorsodorsal pathway associated with online sensorimotor control (Rizzolatti and $\mathrm{Ma}$ telli, 2003), which consists of bilateral superior parietal lobule (SPL) areas and the dorsal premotor cortex (dPMC). We also found increased activity along the right precentral, postcentral, and supramarginal gyri (Fig. 6A). Conversely, amputees showed greater activity than controls within the ventrodorsal pathway associated with motor planning and action organization (Rizzolatti and Matelli, 2003). This includes lateral occipital cortex, and regions of the inferior parietal lobule (IPL) and ventral premotor cortex (vPMC) (Fig. 6B). In addition, amputees demonstrated greater bilateral activity in the vermis and lateral cerebellar hemispheres. Of particular note is the greater activity for amputees within the left precentral gyrus (ipsilateral to the drawing hand), extending into the central sulcus (putative primary sensorimotor cortex).

\section{Amputees but not controls show performance-correlated activity within the former sensorimotor hand area}

To identify performance-correlated changes in areas of the cortex devoted to sensorimotor control of the former and intact hand, we performed an ROI analysis on normatively defined hand sensorimotor cortex, as derived from an independent sample of healthy adults (see Materials and Methods). Figure $7 A$ shows this ROI overlaid on the network of areas displaying significantly more activity for amputees versus controls (i.e., Fig. 6B). This overlay clearly shows our lefthemisphere normative ROI among the areas with greater activation for amputees than for controls.

Analysis of mean PSC computed within these ROIs revealed a significant main effect of Hemisphere $\left(F_{(1,167)}=34.89, p=\right.$ $0.0006)$, but not Group $\left(F_{(1,167)}=1.3, p=0.292\right)$. Critically, we found a significant interaction effect $\left(F_{(1,167)}=18.15, p<\right.$ 
0.0001); post hoc tests (Tukey's HSD) revealed that the interaction effect arose from a significant difference between groups in the ipsilateral (left) hemisphere $(p<0.0001)$ but not in the contralateral (right) hemisphere ( $p>0.8$; Fig. $7 B)$.

Figure $7 C$ illustrates the correlation between left hemisphere PSC and behavioral performance (PDT speed on error-free trials) for each participant. We performed an ANCOVA to assess the difference between the slopes of the two groups' functions. The ANCOVA showed a significant effect of group on the relationship between PSC and behavioral speed $\left(F_{(1,12)}=7.51, p=0.047\right)$. This reflects a stronger behavior dependence in activation of left sensorimotor hand area for amputees compared with controls (i.e., greater slope for amputees than controls in Fig. 7C). Amputees showed a nonsignificant trend toward a positive correlation $(r=0.40, p=0.326)$, whereas controls showed virtually no relationship ( $r=$ $0.11, p=0.801$ ). Power analysis indicated that the PSC-speed correlation among amputees would be significant with 29 participants at the current effect size, whereas the PSC-speed correlation among controls would not reach statistical significance even with 500 participants.

Figure $7 D$ illustrates the correlation between right hemisphere PSC and behavioral endpoint speed. In this right hemisphere ROI, an ANCOVA revealed no significant group effect on the relationship between PSC and speed $\left(F_{(1,12)}=\right.$ 0.07, $p=0.796$; i.e., no between-groups difference in slope). Similar to the left hemisphere ROI, amputees showed a nonsignificant trend toward a positive correlation in the right hemisphere ROI $(r=0.61, p=0.117)$, whereas controls showed a nonsignificant relationship $(r=-0.31, p=$ $0.456)$. Power analysis indicated that the PSC-speed correlation among amputees would be significant with 12 participants at the current effect size, whereas the PSC-speed correlation among controls would not reach statistical significance even with 500 participants.

Together, these findings suggest that: (1) when using their $\mathrm{NDH}$, amputees recruit their ipsilateral (i.e., former) sensorimotor hand area more than do controls; and (2) along with other cortical and subcortical regions, the former sensorimotor hand territory may contribute to the improved control of precision movements with the NDH after chronic forced use.

Effects of amputee characteristics on sensorimotor hand area We found no statistically significant relationships $(\alpha=0.05)$ between activity in the sensorimotor hand ROI during the TaskAny condition and any amputee participant characteristics: phantom vividness, phantom mobility, phantom pain, age, YSA, amputation level, or prosthesis use (Table 1).

\section{Discussion}

This study yielded two major findings. First, when using their NDHs, unilateral amputees performed a PDT significantly faster, more smoothly, and with less time spent in acceleration than did matched controls, approaching levels that would have been expected of their absent DHs. These results are consistent with the hypothesis that chronic forced use of the $\mathrm{NDH}$ leads to substantial improvements of a skill that was previously the exclusive domain of the now-absent DH. Second, this increase in NDH function appears to involve experience-dependent changes in neural control of precision movements. Whereas controls rely heavily on the dorsodorsal (SPL-dPMC) feedback control pathway, amputees show greater recruitment of the ventrodorsal (IPL-vPMC) pathway involved in the internal representation of goal-directed actions. Amputees also engage the normatively defined, former sensorimotor hand representation (ipsilateral to the drawing hand) more than do controls. Although modest, the level of activation within this region shows a greater relationship to performance in amputees versus controls. Below, we discuss these findings and their implications in detail.

\section{Improved performance with chronic forced use of the NDH}

We hypothesized that, if right hand dominance for precision control reflects an intrinsic specialization of the left cerebral hemisphere (Liepmann, 1905; Geschwind et al., 2002; Sainburg, 2005; Shabbott and Sainburg, 2008), then amputees' $\mathrm{NDH}$ performances might improve as a result of chronic 

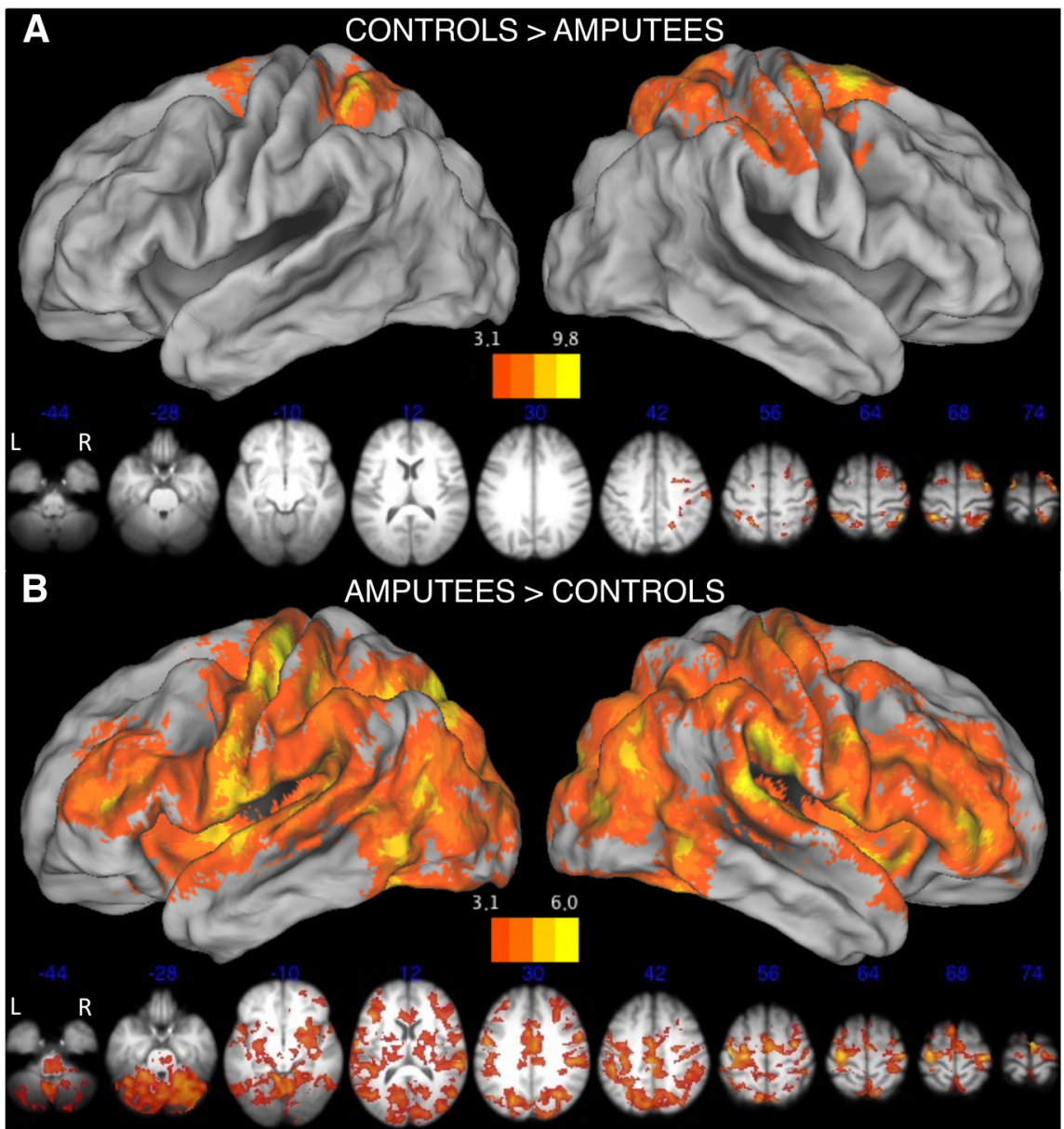

Figure 6. Differences in neural activity between groups, associated with PDT task (relative to Vision-Control baseline). Wholebrain analysis group statistical parametric maps overlaid on PALS atlas. $A$, Areas with greater activity for control participants (NDH) compared with amputees, including dorsodorsal (SPL-dPMC) pathway, involved in feedback control. B, Areas with greater activity for amputees compared with control participants (NDH), including ventrodorsal (IPL-vPMC) and lateral and medial prefrontal regions, areas associated with cognitive control of goal-directed actions. kinematics (Siebner et al., 2002). In contrast to the current study, this earlier work used a handwriting task that lacked a means of quantifying errors and also involved repetitively producing the same word.

Although we lack longitudinal data on these patients, the most likely interpretation for these effects is that the ability to control high precision movements with the amputees' left hands has increased over time as a result of chronic forced use. Although we found no correlation between behavioral performance and time since amputation, all of our amputee participants were at least 8 years after amputation, allowing a substantial period of NDH forced use for all participants. It seems unlikely that unilateral deafferentation/deefferentation, in and of itself, contributes to increased performance of the intact limb. Although there is evidence that acute, reversible unilateral deafferentation can transiently facilitate some sensory (Werhahn et al., 2002b; Björkman et al., 2009) or motor (Björkman et al., 2004) functions of the unaffected hand, these improvements are quite small in magnitude and have not been detected in amputees (Vega-Bermudez and Johnson, 2002).

Forced use may improve NDH performances either through new learning, enhanced intermanual transfer of formerly DH skills, or a combination. More work is required to disambiguate these possibilities. forced use but should never reach the level of controls' DH performances. We found that amputees' error rates were comparable with the NDHs of controls and higher than controls' DHs (Fig. 3E). In contrast, movement speed (Fig. 3B) was equivalent to matched controls' DHs and significantly better than controls' NDHs. A major factor underlying this effect is that amputees' NDH movements are substantially smoother than those of controls and indeed are equivalent to controls' DHs (Fig. $3 C$ ). Healthy right-handed adults exhibit a substantial DH advantage for smoothness that has been attributed to left-hemisphere specializations for the control of intersegmental limb dynamics (Sainburg, 2005). Amputees also spend a smaller fraction of their movement time accelerating compared with controls using their $\mathrm{NDH}$, statistically indistinguishable from the level of controls using their DHs (Fig. 3D). The ability to control acceleration time by controlling muscle timing appears to depend on specializations of the right hemisphere in right-handers (Schaefer et al., 2007). Therefore, our pattern of kinematic results suggests that exclusive and chronic forced use of the left NDH leads to improvements in movement speed by exploiting aspects of control that may be specializations of both the dominant left (smoothness) and nondominant right (acceleration time) cerebral hemispheres. These behavioral results complement evidence showing no significant differences between native right-handers and converted left-handers in writing
Improved NDH performance is supported by a transition from reliance on the dorsodorsal to the ventrodorsal pathway When participants engaged in precision drawing with the NDH, we discovered that controls and amputees differed in their reliance on functionally and anatomically dissociable subdivisions within the dorsal sensorimotor processing stream (Johnson and Grafton, 2003; Rizzolatti and Matelli, 2003; Glover, 2004). Controls showed greater activity than amputees within the SPL and APMC, key nodes of the dorsodorsal pathway involved in feedback-based control of limb movements. Amputees, by contrast, show greater activity than controls within the lateral occipital cortex, IPL, and vPMC, major nodes of the ventrodorsal pathway implicated in the internal representation of complex, goal-directed behavior. These findings suggest that the dramatic improvement of NDH performance is supported by an experience-dependent shift in neural control. In nonexperts (controls), precision drawing with the NDH relies more heavily on feedback control supported by the dorsodorsal pathway (Fig. 6A). With extensive training, experts (DH amputees) come to depend more on the ventrodorsal pathway (Fig. 6B), which supports internal representations of skilled, goal-dependent actions. Furthermore, amputees showed increased engagement of lateral (Ridderinkhof et al., 2004) and medial (Rushworth et al., 2004) prefrontal regions associated with cognitive control of actions, and in bilateral cerebellum. 
A
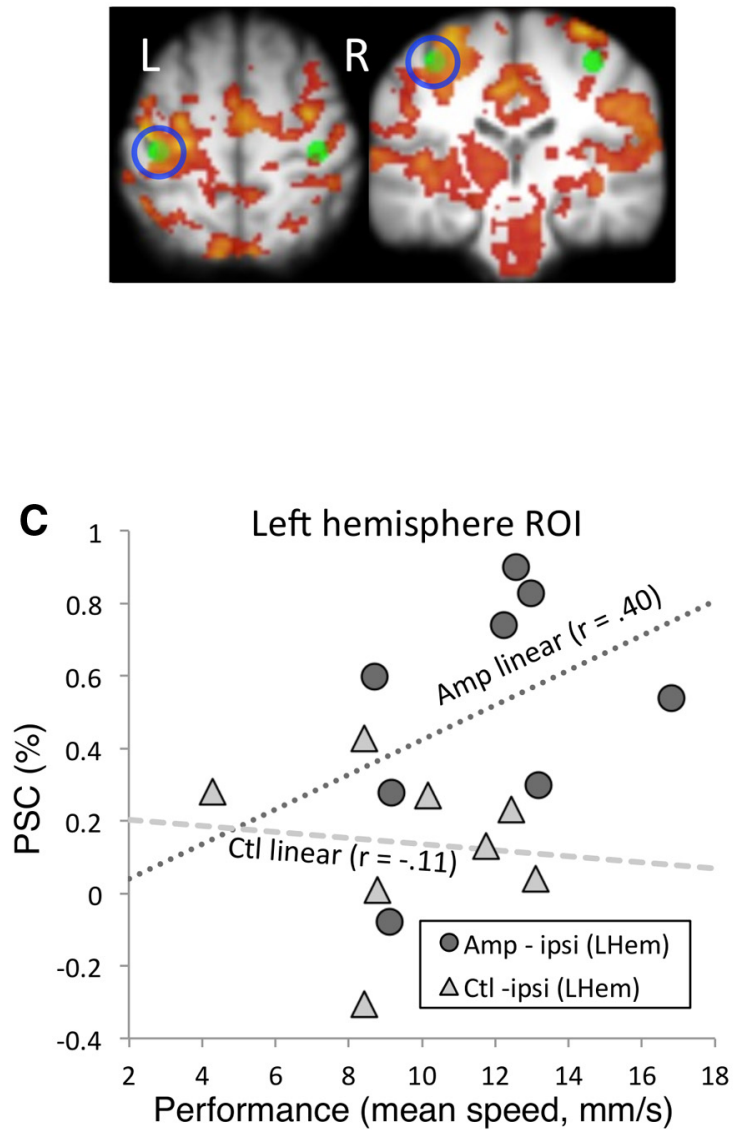

B

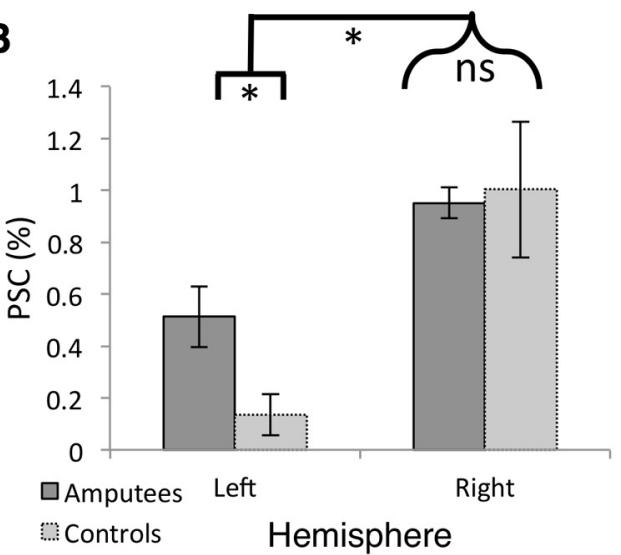

D 2.5

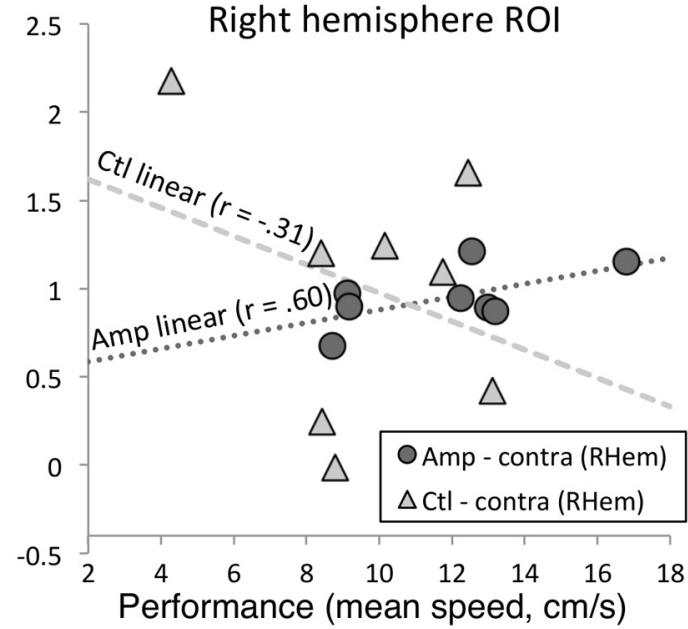

Figure 7. ROI analysis of PSC in normative sensorimotor hand area, showing increased activity in left hemisphere. $A$, ROI location (green), superimposed with amputee-increased activation (Fig. $6 B$ ). Blue circle represents activation in left hemisphere normative ROI. $\boldsymbol{B}$, PSC group mean \pm SEM, demonstrating group effect for left hemisphere (ipsi-movement) only. * Significant difference ( $p<0.001$, Tukey's HSD). C, Relationship between performance (endpoint speed) and left hemisphere (former hand territory) PSC for each participant. Slopes of linear fits are significantly different $(p=0.047$, ANCOVA). D, Relationship between performance and right hemisphere (intact hand territory) PSC for each participant. Slopes of linear fits not significantly different (ns; $p=0.796)$.

Precision drawing by amputees is associated with increased recruitment of the normatively defined former sensorimotor hand territory

Consistent with earlier work (Kew et al., 1994; Hamzei et al., 2001), we detected increased activity within sensorimotor cortex ipsilateral to the drawing hand (Fig. $7 B$ ). Likewise, these increases occurred specifically within the normatively defined former sensorimotor hand territory (Bogdanov et al., 2012). We predicted that activity within the former hand territory would be related to performance on the PDT if this is recruitment is a functional adaptation that supports improved precision movements with the NDH (Frey et al., 2008; Bogdanov et al., 2012). We found a nonsignificant positive relationship between activity in the former hand territory and NDH PDT performance (Fig. 7C) for amputees $(r=0.40)$, but not controls $(r=-0.11)$. Furthermore, the difference between performance-correlation in amputees versus controls achieved significance (Fig. $7 C$, slopes; $p=0.047$ ), suggesting that the ipsilateral hand territory may play a functional role in improved performance after chronic forced use of the NDH. This finding dovetails with our kinematic results suggesting that improvements in amputees' performances include experience-dependent changes in smoothness, a control parameter associated with the left cerebral hemisphere (Sainburg, 2005). Like left-handed adults forced to switch from left-handed to right-handed writing in childhood, we also find greater bilat- eral engagement of parietal and premotor regions when writing with the NDH (Siebner et al., 2002). Converted left-handers show contralateral left sensorimotor activity during writing, but not increased use of the ipsilateral right sensorimotor cortex. By contrast, in addition to contralateral activity, amputees do show increased engagement of the ipsilateral (left) sensorimotor cortex when using the NDH, and this does tend to be related to performance. Together, these results could indicate that, regardless of hand dominance, or the side involved in chronic training, the left sensorimotor cortex may play a key role in experience-dependent improvements in NDH skills.

These data should be interpreted with caution until the completion of further work with larger sample sizes. However, these results are generally consistent with previous studies that have demonstrated a functional role for ipsilateral motor cortex in NDH motor learning (Grafton et al., 2002; Schambra et al., 2011), and with prior speculation about the possible bilateral motor cortex recruitment to support NDH control after dominant limb amputation (Sainburg, 2010). If we are correct, then this reflects an important and heretofore overlooked form of adaptive reorganization of the former hand territory after chronic forced use.

In conclusion, we took advantage of the unique opportunity presented by chronic $\mathrm{DH}$ amputees to investigate the potential for NDH compensation. Our results indicate that, over years or decades of exclusive forced use, the speed, smoothness, and ac- 
celeration profile of precision drawing with the nondominant left hand can achieve levels comparable with what would be expected with the dominant right hand, whereas error rates remained as expected for the NDH. In this respect, performance asymmetries in endpoint control appear highly malleable even in mature adults. These compensatory changes in behavior are supported by greater involvement of the ventrodorsal pathway and lateral (Ridderinkhof et al., 2004) and medial (Rushworth et al., 2004) prefrontal regions implicated in the cognitive control of action. This may reflect an experience-dependent transition from heavy reliance on feedback control to greater reliance on cognitive control. Likewise, the former sensorimotor hand territory shows greater recruitment in amputees and may show more behaviorcorrelated activity in amputees than in controls. These results suggest that intensive training may enable behavioral compensation after loss of $\mathrm{DH}$ function, which may dramatically improve recovery for patients who experience $\mathrm{DH}$ impairment resulting from injury or disease.

\section{References}

Björkman A, Rosén B, van Westen D, Larsson EM, Lundborg G (2004) Acute improvement of contralateral hand function after deafferentation. Neuroreport 15:1861-1865. CrossRef Medline

Björkman A, Weibull A, Rosén B, Svensson J, Lundborg G (2009) Rapid cortical reorganisation and improved sensitivity of the hand following cutaneous anaesthesia of the forearm. Eur J Neurosci 29:837-844. CrossRef Medline

Bogdanov S, Smith J, Frey SH (2012) Former hand territory activity increases after amputation during intact hand movements, but is unaffected by illusory visual feedback. Neurorehabil Neural Repair 26:604-615. CrossRef Medline

Coren S, Porac C (1977) Fifty centuries of right-handedness: the historical record. Science 198:631-632. CrossRef Medline

Elbert T, Rockstroh B (2004) Reorganization of human cerebral cortex: the range of changes following use and injury. Neuroscientist 10:129-141. CrossRef Medline

Frey SH, Bogdanov S, Smith JC, Watrous S, Breidenbach WC (2008) Chronically deafferented sensory cortex recovers a grossly typical organization after allogenic hand transplantation. Curr Biol 18:1530-1534. CrossRef Medline

Geschwind DH, Miller BL, DeCarli C, Carmelli D (2002) Heritability of lobar brain volumes in twins supports genetic models of cerebral laterality and handedness. Proc Natl Acad Sci U S A 99:3176-3181. CrossRef Medline

Glover S (2004) Separate visual representations in the planning and control of action. Behav Brain Sci 27:3-24; discussion 24-78. Medline

Grafton ST, Hazeltine E, Ivry RB (2002) Motor sequence learning with the nondominant left hand: a PET functional imaging study. Exp Brain Res 146:369-378. CrossRef Medline

Hamzei F, Liepert J, Dettmers C, Adler T, Kiebel S, Rijntjes M, Weiller C (2001) Structural and functional cortical abnormalities after upper limb amputation during childhood. Neuroreport 12:957-962. CrossRef Medline

Jacobs S, Danielmeier C, Frey SH (2010) Human anterior intraparietal and ventral premotor cortices support representations of grasping with the hand or a novel tool. J Cogn Neurosci 22:2594-2608. CrossRef Medline

Johnson SH, Grafton ST (2003) From 'acting on' to 'acting with': the functional anatomy of object-oriented action schemata. Prog Brain Res 142: 127-139. CrossRef Medline

Kew JJ, Ridding MC, Rothwell JC, Passingham RE, Leigh PN, Sooriakumaran S, Frackowiak RS, Brooks DJ (1994) Reorganization of cortical blood flow and transcranial magnetic stimulation maps in human subjects after upper limb amputation. J Neurophysiol 72:2517-2524. Medline

Klöppel S, Vongerichten A, van Eimeren T, Frackowiak RS, Siebner HR (2007) Can left-handedness be switched? Insights from an early switch of handwriting. J Neurosci 27:7847-7853. CrossRef Medline

Liepmann H (1905) Die linke Hemisphaere und das Handeln. Muench Med Wochenschr 49:2375-2378.

Mutha PK, Haaland KY, Sainburg RL (2012) The effects of brain lateralization on motor control and adaptation. J Mot Behav 44:455-469. CrossRef Medline

Oldfield RC (1971) The assessment and analysis of handedness: the Edinburgh inventory. Neuropsychologia 9:97-113. CrossRef Medline
Pawela CP, Biswal BB, Hudetz AG, Li R, Jones SR, Cho YR, Matloub HS, Hyde JS (2010) Interhemispheric neuroplasticity following limb deafferentation detected by resting-state functional connectivity magnetic resonance imaging (fcMRI) and functional magnetic resonance imaging (fMRI). Neuroimage 49:2467-2478. CrossRef Medline

Pelled G, Bergstrom DA, Tierney PL, Conroy RS, Chuang KH, Yu D, Leopold DA, Walters JR, Koretsky AP (2009) Ipsilateral cortical fMRI responses after peripheral nerve damage in rats reflect increased interneuron activity. Proc Natl Acad Sci U S A 106:14114-14119. CrossRef Medline

Porter R, Lemon R (1993) Corticospinal function and voluntary movement. Oxford: Clarendon.

Ridderinkhof KR, van den Wildenberg WP, Segalowitz SJ, Carter CS (2004) Neurocognitive mechanisms of cognitive control: the role of prefrontal cortex in action selection, response inhibition, performance monitoring, and reward-based learning. Brain Cogn 56:129-140. CrossRef Medline

Rizzolatti G, Matelli M (2003) Two different streams form the dorsal visual system: anatomy and functions. Exp Brain Res 153:146-157. CrossRef Medline

Rushworth MF, Walton ME, Kennerley SW, Bannerman DM (2004) Action sets and decisions in the medial frontal cortex. Trends Cogn Sci 8:410417. CrossRef Medline

Sainburg RL (2005) Handedness: differential specializations for control of trajectory and position. Exerc Sport Sci Rev 33:206-213. CrossRef Medline

Sainburg RL (2010) Lateralization of goal directed movements (Ellott D, Khan M, eds), pp 219-238. Champaign, IL: Human Kinetics.

Schaefer SY, Haaland KY, Sainburg RL (2007) Ipsilesional motor deficits following stroke reflect hemispheric specializations for movement control. Brain 130:2146-2158. CrossRef Medline

Schambra HM, Abe M, Luckenbaugh DA, Reis J, Krakauer JW, Cohen LG (2011) Probing for hemispheric specialization for motor skill learning: a transcranial direct current stimulation study. J Neurophysiol 106:652661. CrossRef Medline

Shabbott BA, Sainburg RL (2008) Differentiating between two models of motor lateralization. J Neurophysiol 100:565-575. CrossRef Medline

Siebner HR, Limmer C, Peinemann A, Drzezga A, Bloem BR, Schwaiger M, Conrad B (2002) Long-term consequences of switching handedness: a positron emission tomography study on handwriting in "converted" lefthanders. J Neurosci 22:2816-2825. Medline

Smith J, Frey SH (2011) Use of independent component analysis to define regions of interest for fMRI studies. In: International Society for Magnet Resonance in Medicine (ISMRM) Montreal.

Smith SM, Jenkinson M, Woolrich MW, Beckmann CF, Behrens TEJ, Johansen-Berg H, Bannister PR, De Luca M, Drobnjak I, Flitney DE, Niazy RK, Saunders J, Vickers J, Zhang Y, De Stefano N, Brady JM, Matthews PM (2004) Advances in functional and structural MR image analysis and implementation as FSL. Neuroimage 23 [Suppl 1]:S208-S219.

Thesen S, Heid O, Mueller E, Schad LR (2000) Prospective acquisition correction for head motion with image-based tracking for real-time fMRI. Magn Reson Med 44:457-465. CrossRef Medline

Van Essen DC (2005) A Population-Average, Landmark- and Surface-based (PALS) atlas of human cerebral cortex. Neuroimage 28:635-662. CrossRef Medline

Vega-Bermudez F, Johnson KO (2002) Spatial acuity after digit amputation. Brain 125:1256-1264. CrossRef Medline

Verstynen T, Diedrichsen J, Albert N, Aparicio P, Ivry RB (2005) Ipsilateral motor cortex activity during unimanual hand movements relates to task complexity. J Neurophysiol 93:1209-1222. CrossRef Medline

Vines BW, Cerruti C, Schlaug G (2008) Dual-hemisphere tDCS facilitates greater improvements for healthy subjects' non-dominant hand compared to unihemisphere stimulation. BMC Neurosci 9:103. CrossRef Medline

Werhahn KJ, Mortensen J, Kaelin-Lang A, Boroojerdi B, Cohen LG (2002a) Cortical excitability changes induced by deafferentation of the contralateral hemisphere. Brain 125:1402-1413. CrossRef Medline

Werhahn KJ, Mortensen J, Van Boven RW, Zeuner KE, Cohen LG (2002b) Enhanced tactile spatial acuity and cortical processing during acute hand deafferentation. Nat Neurosci 5:936-938. CrossRef Medline

Woolrich MW, Ripley BD, Brady M, Smith SM (2001) Temporal autocorrelation in univariate linear modeling of FMRI data. Neuroimage 14: 1370-1386. CrossRef Medline

Yancosek KE, Mullineaux DR (2011) Stability of handwriting performance following injury-induced hand-dominance transfer in adults: a pilot study. J Rehabil Res Dev 48:59-68. CrossRef Medline 